\title{
THE COLLINEATIONS OF SPACE.
}

Die Lehre von den geometrischen Verwandtschaften. Dritter Band: die eindeutigen linearen Verwandschaften zwischen Gebilden dritter Stufe. By Rudolf STurm. Leipzig and Berlin, B. G. Teubner, 1909, viii + 574 pp.

The third volume of Professor Sturm's treatise has about the same range of discussion for three dimensions as the preceding ones had for one and two respectively, ${ }^{*}$ the size of the volume being explained by the very full treatment given to systems of correlations. This volume is not provided with a separate index nor a preface, but does contain a glossary of 165 technical words not found in the preceding ones, and the table of contents of all four volumes.

The first chapter (pages 1-352) is concerned with the ordinary problems of collineation and correlation in space, being somewhat similar to the treatment found in the corresponding parts of Reye's Geometrie der Lage, but much more extensive. The properties of central and axial perspective, affinity, similarity, and congruence are first brought out to discuss the collineations and polarity defined by linear complexes. All of this leads up to the theorem that a general collineation can be resolved into three axial involutions.

The two kinds of collineation which leave a given quadric invariant, and in particular a space cubic curve are given thirty pages. This could have been materially shortened by a more liberal use of analytic methods, $\uparrow$ the development being almost exclusively synthetic. In fact the need of still another treatise, having the same general content as this first chapter, but proceeding algebraically, is keenly felt. It could be regarded as an appendix to the work of Professor Sturm.

Metrical, particularly focal, properties follow. Here a number of results are necessarily stated in algebraic form, but mostly without proofs. A knowledge of projective analytic geometry of quadrics is presupposed. Besides lines of curva-

\footnotetext{
* See the reviews in volume 15 of the Bulletin; that of Volume 1 on page 135 , and of Volume 2 on page 252 .

$\dagger$ As for example, that employed by Wiman; "Ueber die algebraischen Curven von den Geschlechtern $p=4,5$, and 6, welche eindeutige Transformationen in sich besitzen," Bihang till Kongl. Svenska Vetenskaps-Akad. Handlingar, 1895, and particularly, Miss Van Benschoten; "Birational transformations of algebraic curves of genus four," Amer. Jour. Math., vol. 31 (1909), pp. 213-252.
} 
ture and curves of contact with an enveloping developable, geodesics on a quadric are defined and shown to be covariant in the collineations of a system of confocal quadrics. A few general theorems concerning the commutativity of two collineations are followed by a more thorough discussion of groups generated by central harmonic homologies. Every axial involution can be generated in this way. Cyclic collineations and cyclic correlations of degree four are treated at length.

Over sixty pages (287-352) are devoted to the discussion of groups of collineations. The first topic is that of collineations in inscribed tetrahedral position, i. e., such that a tetrahedron exists having the property that the image of each vertex lies in the opposite face. It is shown that the existence of one such tetrahedron insures the existence of $\infty^{9}$ of them, three vertices being arbitrary. The article on cyclic collineations with nonplanar cycles distinguishes between cycles of odd and of even period, and discusses those of period 4,5 , and 6 at length. These themes have been exhaustively treated in a number of recent dissertations from Breslau and Strassburg.*

In case of period six the principal distinction is between four imaginary invariant points on the one hand, and two real and two imaginary ones on the other. In the article on the general group concept a number of properties are mentioned, but the fundamental question, under what conditions are two collineations commutative, is not touched. $\uparrow$

Besides the regular body groups, the $G_{32}$ defined by six linear complexes mutually in involution, and its linear subgroup of order 16 are treated in detail. The chapter closes with a comprehensive synthetic treatment of the configuration $\left(12_{6}, 16_{3}\right)$ composed of 12 planes, 12 points and 16 lines, such that each of the 16 lines lies in three of the planes and passes through six points, and every point lies in six planes, defining a desmic

\footnotetext{
* Particularly H. Küppers, “ Kollineationen, durch welche fünf gegebene Punkte des Raumes in dieselben fünf Punkte transformiert werden" (Münster, 1890,79 pp.) ; H. Reim, "Wie müssen zwei projektivische Punktfelder aufeinander gelegt werden, damit entsprechend kongruente Polygone cyclisch zusammenfallen?" (Breslau, 1879, 28 pp.) ; R. Krause, "Ueber senäre cyklische Kollineationen"' (Strassburg, 1903, 58 pp.) ; E. Gässler, "Ueber senär cyklische Korrelationen in der Ebene und im Raume" (Strassburg, 1903, 39 pp. ) ; J. Cordier, "Gruppe von 96 Kollineationen (Strassburg, 1905, 58 pp.).

f The theorems given by Reye regarding two commutative linear substitutions are only partly correct. The theorem of Stephanos for binary fields can be readily extended to any number of dimensions, but other forms can also appear in space of three dimensions.
} 
system. This system belongs to a group of 2304 collineations and correlations, which contains subgroups of order 1152 and of order 576. The simple group of collineations of order 168 first discovered by Klein, and that of order 360 found by Valentiner are mentioned only in a footnote. Notwithstanding the excellent discussion in the present treatise and the many valuable memoirs mentioned in it, there is still much important work to be done before the theory of linear and birational transformations can be fully translated into the language of substitutions.

The second chapter (pages 353-574) is concerned with linear systems of curves and surfaces, singular collineations, problems in enumerative geometry and linear systems of linear transformations. It begins with a system of plane curves having one degree of freedom, then two, etc., each successive case being generated by the fan formation from the preceding one. While this treatment is an excellent one, it could be much improved or at least its results made more useful for others by the addition of an algebraic formulation of both processes and results. The section on the generation of curves and surfaces by means of projective pencils follows Cremona somewhat, but is more readable and much more extensive. The treatment of the important subject of restricted systems is more systematic but less extensive than that found in Salmon's Algebra, the only other book containing it. The theory of poles and polars is essentially that of Cremona, but here again, is much longer. Incidentally a generous synthetic treatment of covariant curves and surfaces including the Hessian, the Steinerian, the Cayleyan, and their Jacobian is introduced.

The long sections on singular correlations and collineations begin with the. adjustment of two correlative spaces into a polar space. As first singular form appears the polarity with regard to a cone, or its dual, a conic, and a pair of planes or a pair of points. If now the superposed position of the two spaces be dissolved, and with it the involutorial correspondence, we have the three singular correlations, called by Hirst the central, the planar, and the axial. A well-known special case of the last named is the reciprocity in a special linear complex. By dualizing one of the spaces we obtain the singular collineations of the first kind, but only two forms, as the first and second are really equivalent. A central homology with invariant 0 furnishes an example of the first kind (ordinary artist's perspective) 
and an axial collineation with parameter 0 is an illustration of the second kind.

From this foundation it is now possible to reduce the successive cases to depend on those of singular plane collineations which were treated at length in Volume 2. But complete enumeration is a hopeless task ; after allowing for all possible duplications, over 4000 signatures exist, each one deserving some attention.

The results of this investigation are employed to determine the number of conditions imposed by homology, axial involution, affinity, etc., and then in turn the possible singular transformations in these various types. Linear systems of space correlations, of polar spaces and duality in a pencil of linear complexes are then treated, and the singular and degraded cases included among them are determined.

The idea of apolar linear systems of space correlations leads to a number of new configurations, and serves to systematize a number of theorems found by Rosanes, Reye, Voss, Kohn, and. others.

Given two correlations $C, \Gamma$ having the property that one tetrahedron $\alpha^{\prime} \beta^{\prime} \gamma^{\prime} \delta^{\prime}$ in the space $\Sigma^{\prime}$ exists such that its image $A_{1} B_{1} C_{1} D_{1}$ in $\Gamma$ is inscribed in its image $A B C D$ in $C$; in the two spaces $\Sigma, \Sigma^{\prime}$ there are then $\infty^{9}$ tetrahedra having this property; by performing the operations in inverse order (the $C$ polar of the $\Gamma$ polar) we have $\infty^{9}$ tetrahedra circumscribed about the given ones. The correlations $\Gamma$ and $C$ are called apolar, $C$ is said to support $\Gamma$ and $\Gamma$ to lie in $C$.

If $C_{1}, C_{2}$ are two correlations, each of which supports $\Gamma$, then every correlation of the pencil determined by them will also support it. Similarly for nets, webs, and in general $k$-fold systems. Simultaneously $\Gamma$ may be any correlation of an $i$-fold system, such that $i+k=14$. This idea is now applied to a singular correlation, i. e., $\Gamma$ is now polarity with regard to a cone having its vertex at $S$, and $S^{\prime}$ the center in $\Sigma^{\prime}$. The points $S, S^{\prime}$ are conjugate in $C$. It now follows that every pencil of correlations contains one which supports a given one.

The same procedure is then carried out for collineations, by dualizing one of the spaces and applying $\left(S, s^{\prime}\right),\left(S^{\prime}, s\right)$ connexes as was done in the second volume. Finally a long discussion (57 pages) is given to linear systems of projective configurations which support each other.

Notwithstanding the size of the volume many topics are 
treated very briefly, and some knowledge of various special operations is presupposed. A familiarity with the preceding volumes would not be sufficient preparation for the intelligent reading of the present one.

VIRGIL SNyder.

\section{A SYNOPTIC COURSE FOR TEACHERS.}

Elementarmathematik vom höheren Standpunkte aus. Von F.

Krems. Teil I : Arithmetik, Algebra, Analysis. Vorlesung gehalten im Wintersemester $1907-08.5+590$ pp. Teil II :

Geometrie. Vorlesung gehalten im Sommersemester 1908.

$6+515$ pp. Ausgearbeitet von E. Hellinger. Autogr.

Leipzig, in Kommission bei B. G. Teubner, 1908-09.

THE volumes under review contain a course of lectures intended for prospective teachers of mathematics in the secondary schools of Germany. The objects of the course and the reasons for giving it are so well stated in the introduction to the first volume and are of such vital interest in their application to conditions in our own country, that it seems desirable to quote at length.

"In recent years" - thus does Professor Klein begin his first lecture - "a widespread interest has developed among university teachers of mathematics and the natural sciences regarding the proper training of teachers for our secondary schools. This movement is of quite recent date; for a long period previously our universities were concerned exclusively with the higher science without any reference to the needs of the secondary schools and, in fact, without attempting to bring about a connection with secondary mathematics. But what is the result of such a practice? The young student at the outset of his university work is brought face to face with problems that do not serve to remind him of what he has previously studied and naturally he proceeds to forget all of it quickly and thoroughly. On the other hand, if after leaving the university he enters upon his work as a teacher, he is required to give instruction in the established courses in elementary mathematics and, as he is unable without assistance to bring his new work into relation with his advanced mathematics, he soon adopts the old traditional methods and his university studies become merely a more or less pleasant memory which has no influence on his teaching. 